\title{
Structural biology scales up
}

Understanding the proteome is a hot research topic these days. Activities in this area are often reported in high profile publications and widely covered in scientific news media. Proteomic research is extremely well funded, both from public and private sources. One such example is the Protein Structure Initiative at the National Institutes of Health in the US, which now provides more than $\$ 40$ million annually to support nine research centers in the country.

While large grants have generated excitement and stimulated research activity in the field of structural genomics, a different approach is being developed to study the proteome. In this approach, high resolution structures of individual proteins determined by X-ray (and/or NMR) are fit into medium-tolow resolution envelopes of large molecular assemblies reconstructed from electron microscopic images; the results provide detailed views of the structures and extend our understanding of the biological function of these complexes. The development and progress of this approach was the topic of a recent meeting (see meeting review on page 414 of this issue).

The development of this 'hybrid' approach is important and timely. Structural genomics research focuses on high throughput structure determination and is expected to churn out $\sim 1,000$ new structures each year for the next 10 years. Many of these structures are likely to be domains of long polypeptide chains or subunits of larger complexes. Thus, a large amount of detailed structural information will quickly accumulate, but translating this structural information into a biological or functional context may not be so straightforward. Whereas envelopes of large molecular assemblies reconstructed from EM studies provide gross structural features, they alone are often insufficient to interpret how individual components fit together or contribute to function.

At the other end of the spectrum, although technological advance has made it feasible to determine high resolution structures of increasingly larger molecular machines by X-ray crystallography, there will no doubt be limitations. For example, large complexes in the cell are dynamic entities; they may undergo large scale movement to accomplish functions, or they may rapidly assemble/disassemble during functional cycles. Such particles are unlikely to crystallize easily. Even if they crystallize, they may not diffract well to yield a high resolution structure. In contrast, electron microscopy can be used to study these dynamic states. By combining results from such studies with structures of individual components, the hybrid approach may be the only way - at least for now - to obtain detailed information about these complexes, and will likely become an essential tool for structural biologists.

The hybrid approach is gaining popularity and has already been used to study several systems, including the chaperonin GroEL-GroES complex, the ribosome and the structures of several viruses. Each of these studies provides unique structural insights and significantly advances our understanding of the structure-function relationship of these systems. At Nature Structural Biology, we are excited to see the field mature and take flight. While we have published several papers in this area, we certainly hope to see more work appear on the pages of our journal. 